\title{
In situ synthesis of polyisoprene/grafted single-walled carbon nanotube composites
}

\author{
Liqiang Cui ${ }^{1,2}$, Junsheng $\mathrm{Yu}^{1}$, Xinge $\mathrm{Yu}^{1}$, Yinghai $\mathrm{Lv}^{2}$, Guijiang $\mathrm{Li}^{2}$ and Shixue $\mathrm{Zhou}^{2}$ \\ Polyisoprene (PIp)/single-walled carbon nanotube (SWNT) composites were prepared by grafting PIp from the surface of SWNTs \\ via in situ anionic polymerization using sec-butyllithium as an initiator. The anionic initiator molecules were covalently attached \\ to the SWNTs to form SWNTs-bearing carbanions, as confirmed by Fourier transform infrared analysis and Raman spectra. \\ Scanning electron microscopy (SEM) and transmission electron microscope (TEM) were used to image the PIp-g-SWNT \\ composites; the results show a relatively uniform polymer phase present on the surface of individual, debundled nanotubes. \\ The carbanions on the SWNT surface help separate the nanotubes in solution and lead to the development of homogeneous PIp \\ rubber composites with well-dispersed nanotubes. Compared with pure PIp, the PIp/SWNT nanocomposites showed a significant \\ improvement in thermal properties.
}

Polymer Journal (2013) 45, 834-838; doi:10.1038/pj.2012.225; published online 26 December 2012

Keywords: nanocomposites; polyisoprene; carbon nanotube; property

\section{INTRODUCTION}

Since their discovery, ${ }^{1}$ carbon nanotubes have led to the development of new nanotechnologies because of their exceptional stiffness and strength, and remarkable thermal and electrical properties. ${ }^{2-5}$ Singlewalled carbon nanotubes (SWNTs) have the greatest tensile modulus and tensile strength of any known material. ${ }^{6}$ The Young's modulus of carbon nanotubes can reach $1 \mathrm{TPa},{ }^{7}$ whereas their strength has been measured to reach up to $63 \mathrm{GPa}^{8}$ This has allowed for the fabrication of materials such as super-tough polymer-nanotube composite fibers. ${ }^{9}$

However, carbon nanotubes are very difficult to disperse in polymeric matrices due to their large surface areas and van der Waals forces, which can lead to the formation of strongly bound nanotube aggregates. ${ }^{10,11}$ Various methods to obtain homogeneous and fine dispersions of SWNTs in polymer are currently being used, including noncovalent and covalent functionalization. ${ }^{12-17}$ Noncovalent functionalization methods include surfactant modification, polymer wrapping and polymer absorption. The types of surfactants and polymers used in this method are limited, although the advantage is that the structure and electronic properties of nanotubes are not altered. Covalent functionalization includes the modification of carboxylic groups on SWNTs or the direct introduction of initiators onto the sidewalls of SWNTs. Most methods can disrupt the original structure of nanotubes. Viswanathan et al. ${ }^{18}$ introduced carbanions onto the surface of SWNTs by treating with sec-butyllithium and synthesized polystyrene-attached full-length pristine SWNTs. Chen et al. ${ }^{19,20}$ used the same method to graft poly(tert-butyl acrylate) and poly(tert-butyl acrylate)-b-poly(methyl methacrylate) onto the surface of SWNTs.

However, this method is rarely used for rubber polymerization. As conventional rubbers are basically synthesized by anionic polymerization, which requires very harsh reaction conditions and synthesis techniques, it is difficult to realize the in situ polymerization of rubber and carbon nanotubes. This hinders the development of the synthesis of rubber/CNT composites by in situ polymerization; thus, few reports address this technique. Through this study, we have taken the lead in developing in situ anionic polymerization and have successfully synthesized polyisoprene (PIp) rubber/SWNT composites. Carbon nanotubes with surfaces featuring covalently attached PIp were efficiently dispersed in a PIp rubber matrix. Additionally, we investigated the morphology and solubility, as well as thermal stability, of PIp/SWNT nanocomposites.

\section{EXPERIMENTAL PROCEDURE}

Materials

The SWNTs (purity $>90$ weight $\%$, length $5-30 \mu \mathrm{m}$, specific surface area $407 \mathrm{~m}^{2} \mathrm{~g}^{-1}$ ) used in this study were supplied by Chengdu Organic Chemical Co. (Chengdu, China). They were synthesized by chemical vapor deposition and measured $\sim 1-10 \mathrm{~nm}$ in diameter. To ensure that the nanotubes were free of air and absorbed moisture prior to functionalization, they were dried under vacuum at $180^{\circ} \mathrm{C}$ for $24 \mathrm{~h}$ and subsequently stored in argon. Anhydrous cyclohexane and sec-butyllithium (1.4 M in cyclohexane) were purchased from

${ }^{1}$ State Key Laboratory of Electronic Thin Films and Integrated Devices, School of Optoelectronic Information, University of Electronic Science and Technology of China (UESTC), Chengdu, China and ${ }^{2}$ College of Chemical and Environmental Engineering, Shandong University of Science and Technology, Qingdao, China

Correspondence: Professor J Yu, State Key Laboratory of Electronic Thin Films and Integrated Devices, School of Optoelectronic Information, University of Electronic Science and Technology of China (UESTC), Chengdu 610054, China.

E-mail: jsyu@uestc.edu.cn

Received 4 May 2012; revised 18 August 2012; accepted 4 November 2012; published online 26 December 2012 
Sigma-Aldrich (Shanghai, China) and used as received. Isoprene was purchased from Sigma-Aldrich and was distilled from $\mathrm{CaH}_{2}$ under vacuum.

\section{Preparation of the PIp/SWNT nanocomposites}

SWNT-based PIp nanocomposites were prepared by in situ anionic polymerization in a cyclohexane solution. The SWNTs $(41 \mathrm{mg})$ were placed in a $100-\mathrm{ml}$ flask. The flask was washed with argon gas and subsequently boiled in a gas burner for $2 \mathrm{~h}$ under vacuum. Then, $50 \mathrm{ml}$ of anhydrous cyclohexane was transferred to the flask by a syringe, and the mixture was ultrasonically vibrated for $3 \mathrm{~h}$ to disperse the SWNTs as thoroughly possible. Subsequently, $0.30 \mathrm{ml}$ of $1.4 \mathrm{M} \mathrm{sec}$-butyllithium was introduced into the reactor via a syringe. After the reactor was ultrasonically vibrated for $3 \mathrm{~h}, 10 \mathrm{ml}$ of Ip monomer was introduced into the reactor. The polymerization was kept at $50{ }^{\circ} \mathrm{C}$ for $5 \mathrm{~h}$ under magnetic stirring and then terminated with degassed methanol. The polymer was precipitated and dried under vacuum at $60^{\circ} \mathrm{C}$ for $24 \mathrm{~h}$ to obtain the PIp/SWNT nanocomposites. PIp-grafted carbon nanotubes (PIp-g-SWNTs) were separated from the polymer matrix by refluxing in toluene for $24 \mathrm{~h}$ and repeatedly washed with toluene and finally vacuum filtered through a $0.2 \mu \mathrm{m}$ Teflon membrane. The final product of PIp-grafted SWNTs, free of ungrafted PIp, was dried overnight in vacuum. To remove the unreacted sec-butyllithium, SWNT-initiator samples used for IR and Raman spectral characterization were obtained by washing five times with cyclohexane.

For accurate comparisons, pure PIp rubber was prepared by anionic polymerization using sec-butyllithium as a catalyst; the polymerization conditions were consistent with those used for PIp/SWNT-composites polymerization.

\section{Characterization}

Fourier transform infrared analysis was carried out by using a MAGNA-IR 560 (Nicolet Company, Redwood City, CA, USA) with a resolution of $4.0 \mathrm{~cm}^{-1}$ at room temperature. Raman spectra were measured on a Dilor Labram-1B Raman spectrometer (Dilor Company, Paris, France) using a He-Ne laser at an excitation wavelength of $632.8 \mathrm{~nm}$. Viscosity measurements were performed in a toluene solution with an Ubbelohde capillary viscometer at $30^{\circ} \mathrm{C}$. The average molecular weight was calculated using the Mark-Houwink relation: ${ }^{21}$ $[\eta]=2.0 \times 10^{-2} \mathrm{M \eta}^{0.73}$, where $\mathrm{M \eta}$ is the viscosity-average molecular weight. Scanning electron microscopy observations were performed with a LEO 1455 VP microscope (Carl-Zeiss Company, Oberkochen, Germany). The samples were coated with a thin film of conducting metal $(\mathrm{Au})$ to avoid charging problems during imaging. A TEM, Phillips CM20 (Philips-FEI Company, Eindhoven, the Netherlands), was used to observe the microstructure of the samples, that is, very dilute suspensions in chloroform, at an acceleration voltage of $120 \mathrm{kV}$. The glass transition temperature $\left(T_{\mathrm{g}}\right)$ was determined using a TA2000 differential scanning calorimeter at a heating rate of $10^{\circ} \mathrm{C} / \mathrm{min}$ from $-90-100{ }^{\circ} \mathrm{C}$. Two continuous scanning cycles were performed to eliminate the influence of thermal history; the data obtained from the second scanning were accepted. The thermal decomposition temperature was determined by TGA over a temperature range from $40-900{ }^{\circ} \mathrm{C}$ at a scanning rate of $20^{\circ} \mathrm{C} / \mathrm{min}$ under the protection of $\mathrm{N}_{2}$ flow.

Table 1 Results for isoprene polymerization catalyzed by sec-butyllithium only and with SWNT-initiator

\begin{tabular}{lcccccc}
\hline \multicolumn{7}{c}{ SWNTs } \\
Run. & & SWNT & Conver- & fraction in & $M \eta$ & Extracted by \\
No & Initiator content $(g)$ & sion (\%) & Plp (wt \%) & $\left(\times 10^{-4}\right)$ & Toluene (\%) \\
\hline 1 & I $^{\text {a }}$ & 0 & 92.0 & 0 & 1.82 & 100 \\
2 & SWNT-Is & 0.041 & 60.5 & 1.0 & 2.58 & 79.0 \\
\hline
\end{tabular}

Polymerization conditions: [Initiator] $=7.0 \times 10^{-3} \mathrm{moll}^{-1}, 50 \mathrm{ml}$ cyclohexane as the solvent, $10 \mathrm{ml}$ of Ip as the monomer, temperature $=50^{\circ} \mathrm{C}$ and polymerization time $5 \mathrm{~h}$.

${ }^{a}$ sec-butyllithium as the initiator.

\section{RESULTS AND DISCUSSION}

The anionic polymerization of Ip was carried out in cyclohexane at $50{ }^{\circ} \mathrm{C}$, the results of which are shown in Table 1 . The polymerization of Ip using SWNTs treated by sec-butyllithium (SWNT-Is) as the initiator showed a lower conversion $(60.5 \%)$ than that using the pure initiator (92.0\%), and the molecular weight of PIp in the SWNT composites was greater than that in neat PIp. Anionic polymerization is difficult to control and usually disturbed by inherent side reactions. Thus, the low catalytic activity observed in this polymerization should be correlated with the structure of the carbon nanotubes. The use of a lower initiator content during anionic polymerization can produce a higher-molecular weight polymer.

PIp chains were extracted from the PIp/SWNT composites by refluxing with toluene in a Soxhlet extractor for $24 \mathrm{~h}$. The results show that only a fraction of the PIp was extracted, whereas the neat PIp polymerized with the pure initiator was completely extracted. This indicates that the PIp chains in the SWNT composites were attached to the surface of the SWNTs by the anionic initiator. Thus, the motion of the PIp chains was restrained by the interfacial interaction between the chains and the SWNTs. Undissolved PIp chains attached to the surface of the SWNTs formed the SWNT-g-PIp structure.

The pristine SWNTs, initiator-attached SWNTs, and the polymerfunctionalized SWNTs (SWNT-g-PIp) were examined by Fourier transform infrared spectroscopy, and their spectra are presented in Figure 1. Compared with that of the pristine SWNTs (Figure 1a), the IR spectrum of a SWNT-initiator sample (Figure $1 \mathrm{~b}$ ) shows new signals due to the $\mathrm{C}-\mathrm{H}$ stretching of the sec-butyl groups in the range of $2850-2950 \mathrm{~cm}^{-1}$, after treating with sec-butyllithium. As unreacted sec-butyllithium was removed by washing with cyclohexane, Figure $1 \mathrm{~b}$ indicates that the functionalization of the SWNTs was successful. The peak at $1640-1720 \mathrm{~cm}^{-1}$ in Figure 1c can be assigned to the stretching mode of the $\mathrm{C}=\mathrm{C}$ group, indicating the presence of PIp chains in the SWNT-g-PIp composites, and cannot be removed by extracting with good solvent for PIp. It should be noted that in a control experiment, the mixtures of SWNT and PIp were sonicated for $3 \mathrm{~h}$. Then, the mixtures were filtered and washed using the same protocol described in the experimental section. The free polymer was completely removed from the nanotube residue.

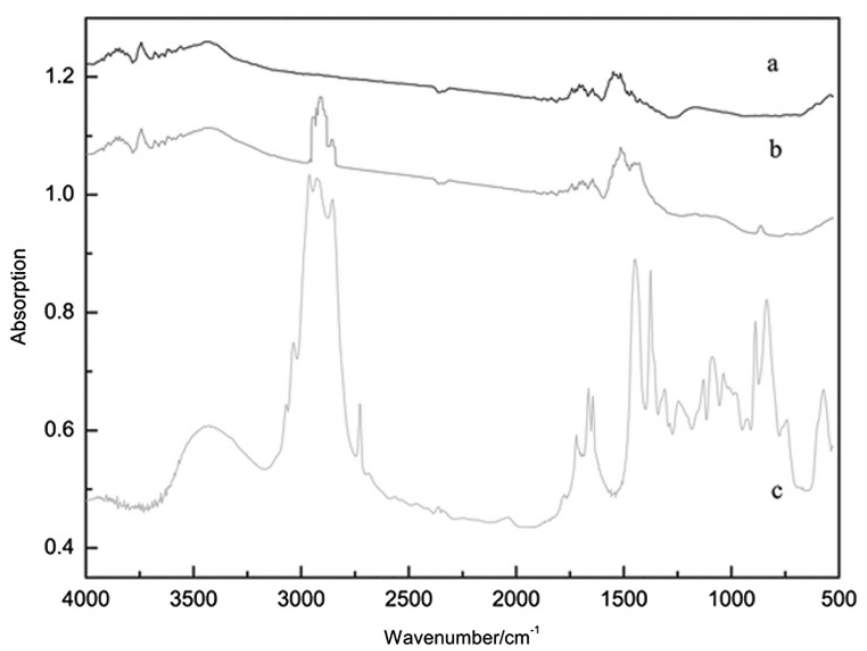

Figure 1 Fourier transform infrared spectra of the pristine SWNTs (a), SWNT-initiator (b) and SWNT-g-PIp (c). A full color version of this figure is available at Polymer Journal online. 
Raman spectroscopy is one of the most useful techniques used to study and characterize nanotubes and also indicate the nature of sidewall functionalization. The graphite-like tangential mode around the G-band $\left(1600 \mathrm{~cm}^{-1}\right)$ and the D-band $\left(1290 \mathrm{~cm}^{-1}\right)$ in a Raman spectrum provides a signature of disorder and/or defects in these structures. $^{22}$ The Raman spectra in Figure 2 show that the secbutyllithium treatment (Figure 2b) and PIp-grafted SWNTs (Figure 2c) caused an increase in the relative intensity of the D-band (the $s p^{3}$ mode signal) compared with that of the pristine SWNTs (Figure 2a). This increase is not due to sonication because control samples in which SWNTs and PIp were sonicated in cyclohexane for $3 \mathrm{~h}$ exhibited no change in the disorder mode. The partial destruction of the conjugated structure of the SWNTs' sidewalls due to attack by sec-butyllithium and the chemical modification of the sidewalls were confirmed. Compared with that shown in Figures $2 \mathrm{~b}$ and $\mathrm{c}$, the intensity of the D-band $\left(1290 \mathrm{~cm}^{-1}\right)$ showed no apparent change, indicating that PIp chain growth was initiated at carbanion-bearing sites on the SWNTs.

The detailed morphology and size of the composites were analyzed by SEM and TEM. The microscopic studies showed that the tubular $\mathrm{PIp} / \mathrm{SWNT}$ composites have a core-shell structure, where carbon nanotube cores are encapsulated by a PIp shell. Compared with the carbon nanotube diameter, the diameters of the tubular composites are much larger. As shown in Figure 3a, the pristine SWNTs exist as bundles or ropes, and according to our observations, the diameters of the SWNTs are not completely identical to one another. Figure 3b shows an image of the carbon nanotubes in the PIp matrix. It is also clear that the PIp/SWNTs composites measure $60-200 \mathrm{~nm}$ in diameter. The fracture surface of the composites clearly shows the dispersion and breakage of the nanotubes within the PIp matrix, as well as a thick, uniform layer of PIp coating the surface of the SWNTs. The coaxial structure of the resulting PIp/SWNT composites was also confirmed by TEM observation. This indicates the existence of strong interfacial bonding between the PIp and nanotubes in the polymer composite. Such morphology has been argued to assist the dispersion of the SWNTs in the polymer matrix and thereby form homogeneous nanocomposites. $^{23}$

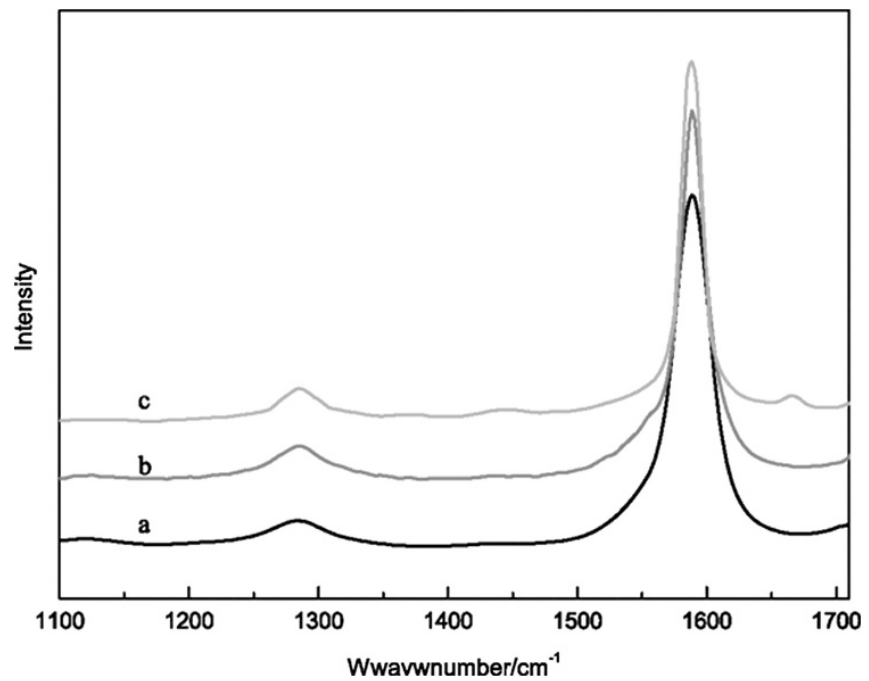

Figure 2 Raman spectra of the pristine SWNTs (a), SWNT-initiator (b) and SWNT-g-PIp(c). A full color version of this figure is available at Polymer Journal online.
The TEM results (Figure 4a) reveal that the pristine SWNTs exist as endless, tangled, hollow ropes with a rather smooth surface. When they are coated with PIp, the morphology of the SWNTs changes remarkably. Figure $4 \mathrm{~b}$ shows the thickened diameter of a single tube compared with the diameters of individual SWNTs, which range from $1-10 \mathrm{~nm}$. The isoprene monomer is uniformly polymerized on the surface of the SWNT due to anionic polymerization initiated by carbanion-bearing SWNTs. The PIp/SWNT composites turn into coaxial core-shell-structured agglomerates, with the polymer particulates coating the nanotubes. The negative charges on the SWNTs undoubtedly result in their debundling; hence, few bundles remain after functionalization with sec-butyllithium. Carbon nanotubes were fully debundled by the mutual electrostatic repulsion between individual nanotubes provided by the carbanions bonded to their surfaces. ${ }^{18,24}$ Moreover, the long, high-power ultrasonic treatment led to the remarkable shortening of the SWNTs. Figure $4 \mathrm{~b}$ shows individually dispersed SWNTs coated with PIp layers. All of the results obtained by IR spectroscopy, SEM and TEM support the notion that PIp can be grafted onto the surface of SWNTs, and pristine SWNTs can be separated into individual tubes by anionic polymerization.

The solubility of PIp/SWNT composites in a $\mathrm{CHCl}_{3}$ solution was also investigated. The SWNTs were very difficult to disperse in $\mathrm{CHCl}_{3}$. Two milligrams of the SWNTs in $10 \mathrm{ml}$ of $\mathrm{CHCl}_{3}$ was sonicated for
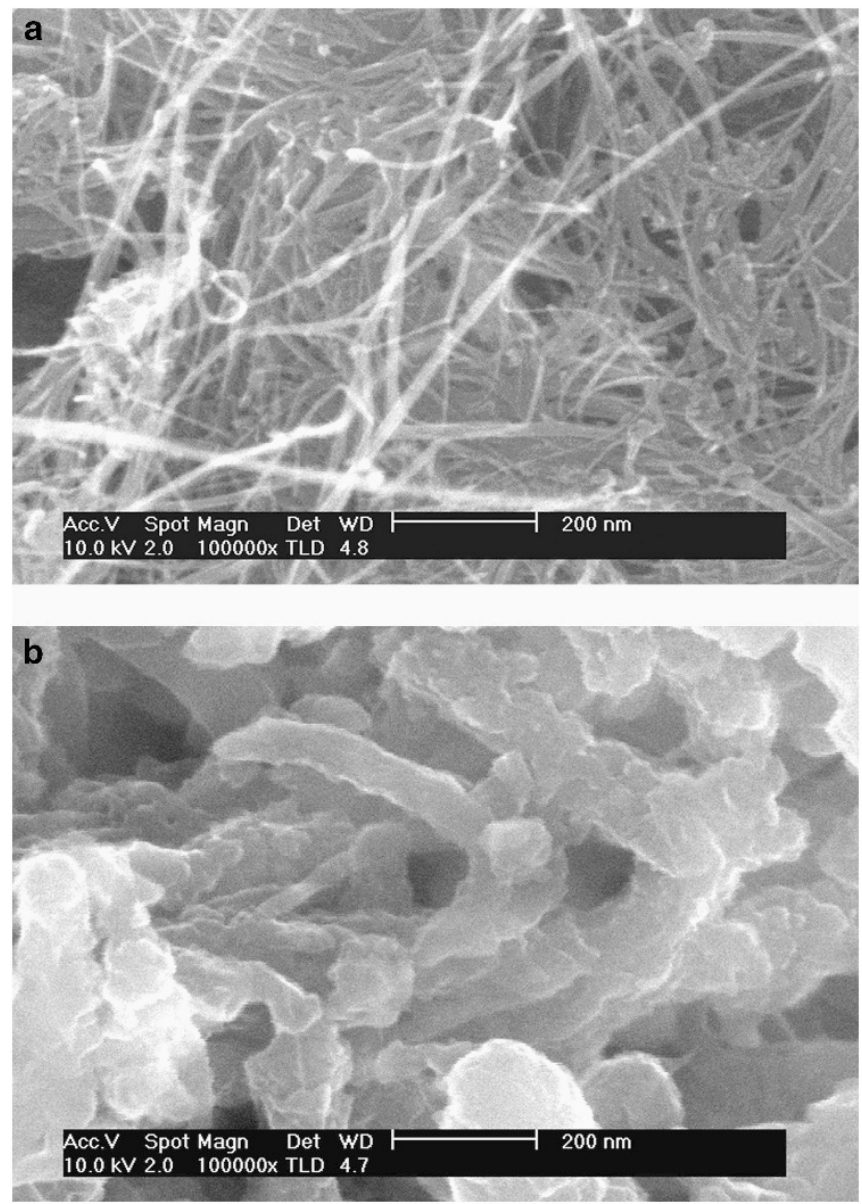

Figure 3 SEM images of pristine SWNTs (a) and PIp/SWNT nanocomposites (1.0 weight $\%$ SWNTs) (b). 

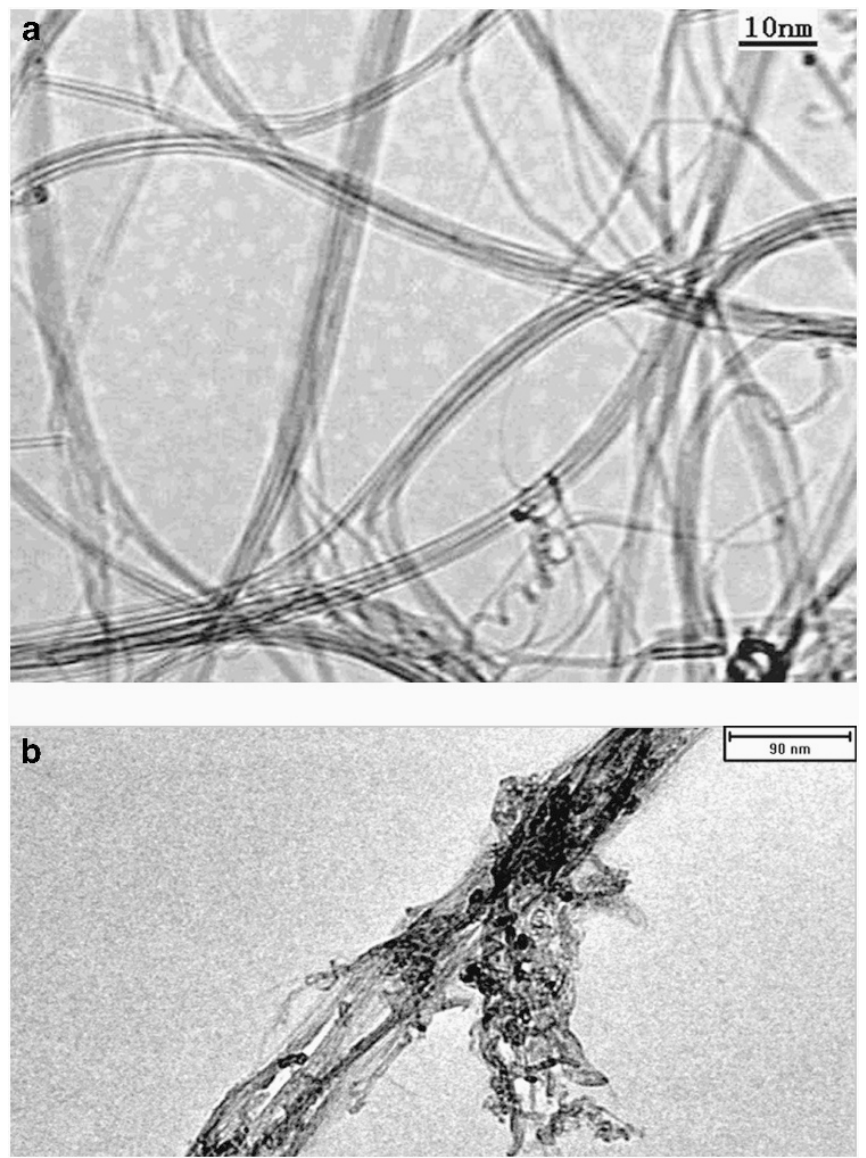

Figure 4 TEM images of pristine SWNT (a) and PIp/SWNT nanocomposites (1.0 weight $\%$ SWNTs) (b).

$60 \mathrm{~min}$, but sedimentation occurred $1 \mathrm{~h}$ after the sonication was halted, as shown in Figure 5a. However, the PIp/SWNT composites were homogeneously dispersed in the $\mathrm{CHCl}_{3}$ solvent. As shown in Figures 5b, $2 \mathrm{mg}$ of the PIp/SWNT composites was dispersed in $10 \mathrm{ml}$ of $\mathrm{CHCl}_{3}$ by sonicating for $60 \mathrm{~min}$; no precipitation of nanotubes from this solution was observed, even after 5 days following sonication. All of the results prove that the PIp/SWNT composites synthesized by anionic polymerization significantly enhanced the solubility of the SWNTs in organic solvents.

The thermal properties of the PIp/SWNT composites were measured by DSC and TGA, the results of which are shown in Figures 6 and 7. The addition of SWNTs increases the $T_{\mathrm{g}}$ values: $T_{\mathrm{g}}$ increases from $-63.7^{\circ} \mathrm{C}$ (pure PIp) to $-56.7^{\circ} \mathrm{C}$ for the PIp/SWNT composites with a SWNT concentration of 1.0 weight $\%$. The $T_{\mathrm{g}}$ value represents the extent of macromolecular segment motion. The strong interaction between the SWNTs and the PIp covalently attached to the nanotubes' surfaces results in a decrease in the mobility of the polymer chains. In effect, the attachment of PIp chains to the SWNTs' surface provides strong interfacial adhesion between the polymer chains and nanotubes, and this confines macromolecular segment motion, resulting in an increase in $T_{\mathrm{g}}$. Figure 7 shows the thermal stability of the PIp/SWNT nanocomposites and pure PIp. The temperature at which the starting material experiences a $5 \%$ weight loss is defined as the thermal decomposition temperature $\left(T_{\mathrm{d}}\right) \cdot{ }^{16,25}$ By comparing the curve of the PIp/SWNT composites $\left(T_{\mathrm{d}}=368.4^{\circ} \mathrm{C}\right)$ with that of pure PIp $\left(T_{\mathrm{d}}=354.0^{\circ} \mathrm{C}\right)$, the thermal stability of the

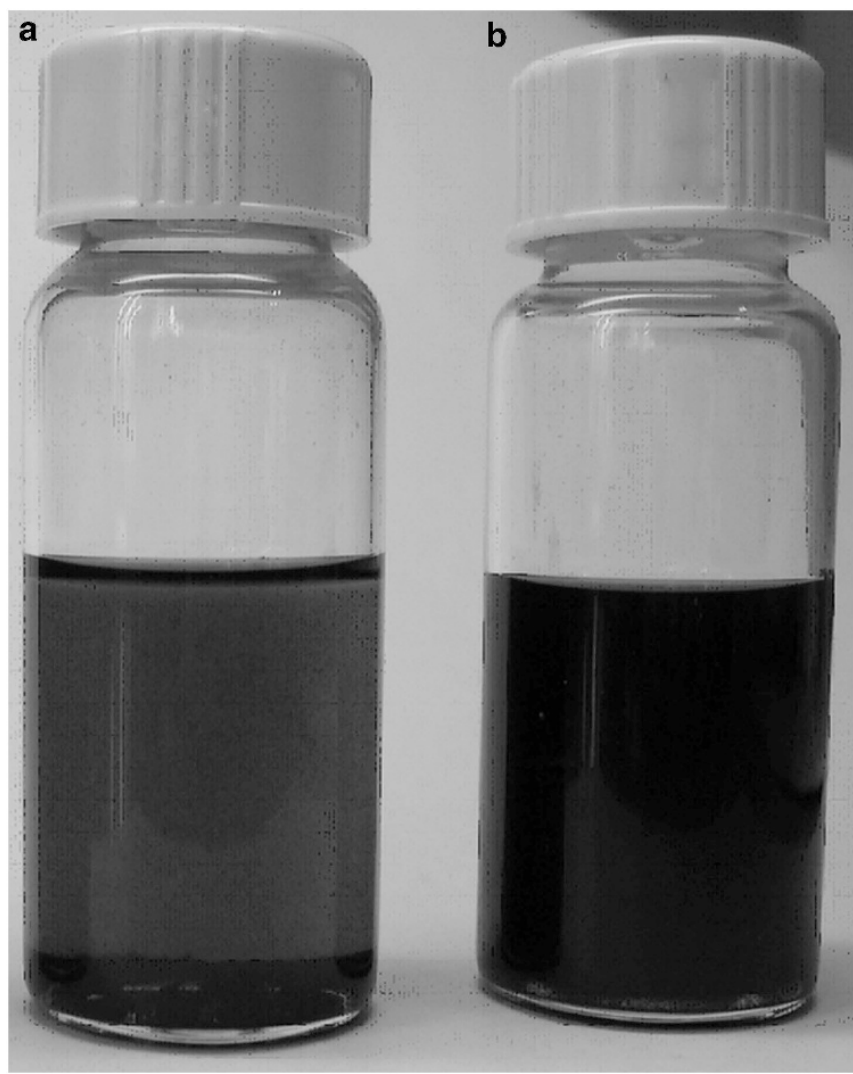

Figure 5 Stability of pristine SWNTs ( $t=1 \mathrm{~h})(\mathrm{a})$ and PIp/SWNT composites (1.0 weight \% SWNTs) ( $t=120 \mathrm{~h}$ ) in $\mathrm{CHCl}_{3}$ (after sonication for $60 \mathrm{~min}$ ) (b). The SWNT content in each of the bottles was $0.2 \mathrm{mg} / \mathrm{ml}$. A full color version of this figure is available at Polymer Journal online.

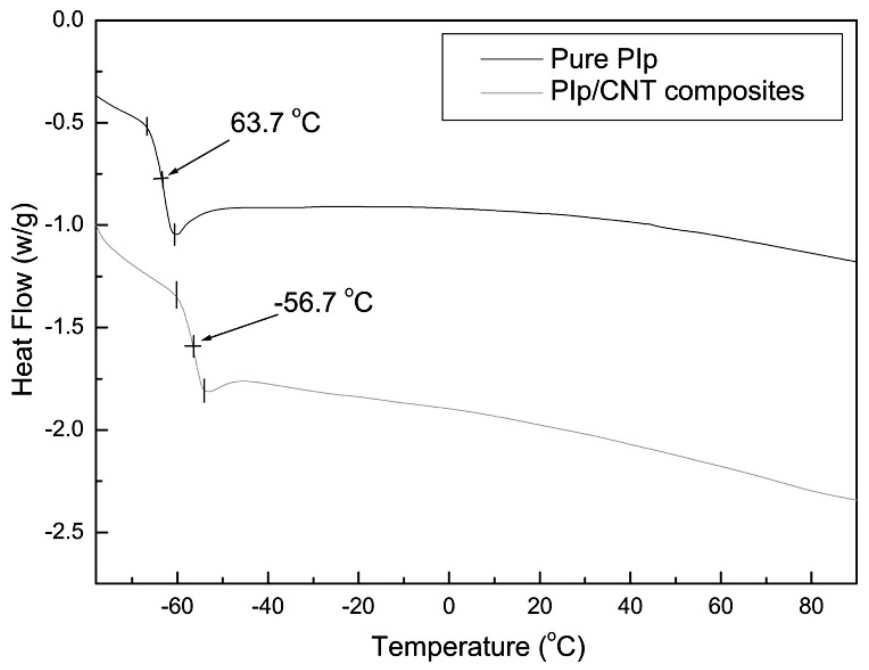

Figure 6 DSC thermograms of pure PIp and PIp/SWNT composites.

nanocomposites shows an increase of $\sim 14.4^{\circ} \mathrm{C}$. This indicates that the covalently bonded PIp-SWNT structures can improve the decomposition temperature of the PIp matrix. The increase in $T_{\mathrm{d}}$ is attributed to the interaction between the polymer chains and SWNT surfaces. 


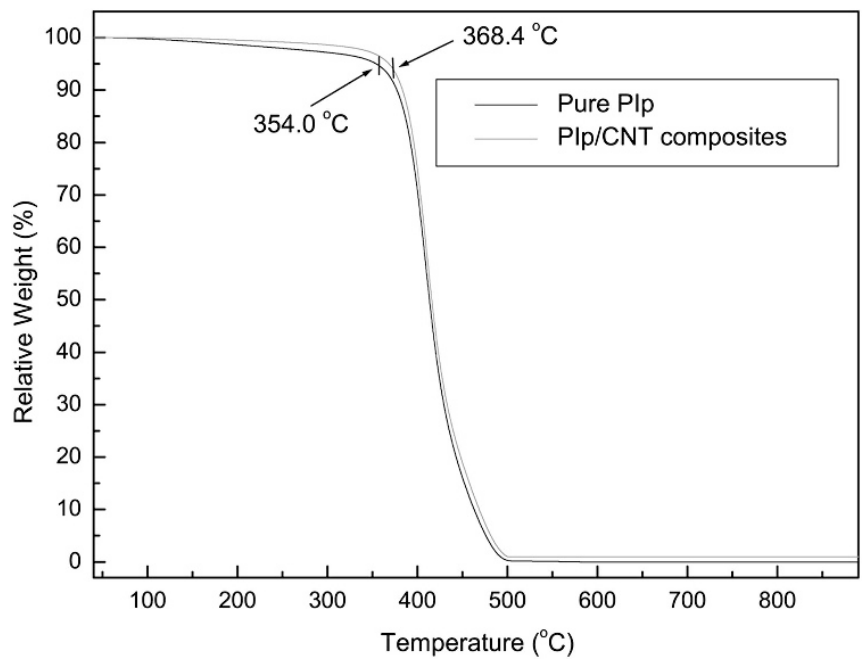

Figure 7 TGA thermograms of the weight loss as a function of temperature for pure PIp and PIp/SWNT composites.

\section{CONCLUSIONS}

SWNTs have been successfully grafted with PIp via anionic polymerization initiated by carbanion-bearing SWNTs. Carbanions were fixed onto SWNTs by treating the SWNTs with sec-butyllithium; PIp chains grew then from these carbanions. The polymer chains were covalently attached to the SWNTs. Owing to the grafting nature of PIp, SWNTs grafted with PIp can be more efficiently dispersed in a PIp matrix. PIp/SWNT composites show better solubility in organic solvents. Moreover, carbon nanotubes strongly interact with the PIp matrix. The results show that the $T_{\mathrm{g}}$ and thermal stability of rubber/SWNT composites clearly increase relative to those of pure rubber polymers. The polymerization of diene monomers on SWNT surfaces by anionic polymerization can provide a new alternative for the fabrication and application of rubber-SWNT nanomaterials.

\section{ACKNOWLEDGEMENTS}

This work was supported by the National Science Foundation of China (NSFC) (Grant No. 61177032), the Foundation of Chunlei Plan of Shandong University of Science and Technology (No. 01100731302) and the Foundation for Innovative Research Groups of the NSFC (Grant No. 61021061).
1 lijima, S. Helical microtubules of graphitic carbon. Nature 354, 56-58 (1991).

2 Ajayan, P. M. Nanotubes from carbon. Chem. Rev. 99, 1787-1799 (1999).

3 Thostenson, E. T., Ren, Z. \& Chou, T. W. Advances in the science and technology of carbon nanotubes and their composites: a review. Compos. Sci. Technol 61, 1899-1912 (2001).

4 Dai, H. J. Carbon nanotubes: synthesis, integration, and properties. Acc. Chem. Res 35, 1035-1044 (2002)

5 Hirsch, A. Functionalization of single-walled carbon nanotubes. Angew Chem. Int. Ed. 41, 1853-1859 (2002).

6 Qin, S. H., Qin, D. Q., Ford, W. T., Resasco, D. E. \& Herrera, J. E. Polymer brushes on single-walled carbon nanotubes by atom transfer radical polymerization of n-butyl methacrylate. J. Am. Chem. Soc. 126, 170-176 (2004).

7 Treacy, M. M. J., Ebbesen, T. W. \& Gibson, J. M. Exceptionally high Young's modulus observed for individual carbon nanotubes. Nature 381, 678-680 (1996).

8 Yu, M., Lourie, O., Dyer, M. J., Kelly, T. F. \& Ruoff, R. S. Strength and breaking mechanism of multiwalled carbon nanotubes under tensile load. Science 287, 637-640 (2000).

9 Dalton, A. B., Collins, S., Munoz, E., Razal, J. M., Ebron, V. H., Ferraris, J. P., Coleman, J. N., Kim, B. G. \& Baughman, R. H. Super-tough carbon nanotube fibres. Nature 423, 703-703 (2003).

10 Shi, S., Zhang, L. \& Li, J. Electrical and dielectric properties of multiwall carbon nanotube/polyaniline composites. J. Polym. Res. 16, 395-399 (2009).

11 Garg, P., Singh, B. P., Kumar, G., Gupta, T., Pandey, I., Seth, R. K., Tandon, R. P. \& Mathur, R. B. Effect of dispersion conditions on the mechanical properties of multi-walled carbon nanotubes based epoxy resin composites. J. Polym. Res. 18, 1397-1407 (2011).

12 Tasis, D., Tagmatarchis, N., Georgakilas, V. \& Prato, M. Soluble carbon nanotubes. Chem. Eur. J 9, 4000-4008 (2003).

13 Niyogi, S., Hamon, M. A., Hu, H., Zhao, B., Bhowmik, P., Sen, R., Itkis, M. E. \& Haddon, R. C. Chemistry of single-walled carbon nanotubes. Acc. Chem. Res. 35, 1105-1113 (2002)

14 Star, A., Stoddart, J. F., Steuerman, D. W., Heath, J. R. \& Stoddart, J. F. Starched carbon nanotubes. Angew Chem. Int. Ed. 41, 2508-2512 (2002).

15 Gomez, F. J., Chen, R. J., Wang, D., Waymouth, R. M. \& Dai, H. Ring opening metathesis polymerization on non-covalently functionalized single-walled carbon nanotubes. Chem. Commun. 190-191 (2003).

16 Peng, H. Q., Alemany, L. B., Margrave, J. L. \& Khabashesku, V. N. Sidewall carboxylic acid functionalization of single-walled carbon nanotubes. J. Am. Chem. Soc. 125, 15174-15182 (2003).

17 Kong, H., Gao, C. \& Yan, D. Y. Controlled functionalization of multiwalled carbon nanotubes by in situ atom transfer radical polymerization. J. Am. Chem. Soc. 126, 412-413 (2004).

18 Viswanathan, G., Chakrapani, N., Yang, H., Wei, B. Q., Chung, H. S., Cho, K. W., Ryu C. Y. \& Ajayan, P. M. Single-step in situ synthesis of polymer-grafted single-wall nanotube composites. J. Am. Chem. Soc. 125, 9258-9259 (2003).

19 Chen, S. M., Shen, W. M., Wu, G. Z., Chen, D. Y. \& Jiang, M. A new approach to the functionalization of single-walled carbon nanotubes with both alkyl and carboxyl groups. Chem. Phys. Lett. 402, 312-317 (2005).

20 Chen, S. M., Chen, D. Y. \& Wu, G. Z. Grafting of Poly(tBA) and PtBA-b-PMMA onto the surface of SWNTs using carbanions as the initiator. Macromol. Rapid Commun. 27, 882-887 (2006).

21 Feng, X. The Study of High Cis-Polyisoprene Synthesized by Anionic Polymerization[D] (Dalian University of Technology, China, 2009).

22 Lefrant, S. Raman and SERS studies of carbon nanotube systems. Curr. Appl. Phys. 2, 479-482 (2002).

23 Gong, X. Y., Liu, J., Baskaran, S., Voise, R. D. \& Young, J. S. Surfactant-assisted processing of carbon nanotube/polymer composites. Chem. Mater. 12, 1049-1052 (2000).

24 Liang, F., Sadana, A. K., Peera, A., Chattopadhyay, J., Gu, Z., Hauge, R. H. \& Billups, W. E. A convenient route to functionalized carbon nanotubes. Nano Lett. 4, 1257-1260 (2004).

25 Pucciariello, R., Villani, V. \& Giammarino, G. Thermal behaviour of nanocomposites based on linear-low-density poly(ethylene) and carbon nanotubes prepared by high energy ball milling. J. Polym. Res. 18, 949-956 (2011). 